\title{
Estudo comparativo da frequência cardíaca obtida por dispositivo oscilométrico e monitor multiparamétrico em cadelas durante ovariossalpingohisterectomia
}

\author{
Francisco Lima Silva ${ }^{1 *}$, Daniel Serafim de Andrade Rodrigues ${ }^{2}$, Catarina Rafaela Alves \\ da Silva ${ }^{3}$, Yago Gabriel da Silva Barbosa ${ }^{4}$, Thiago Sousa da Silva ${ }^{2}$ \\ ${ }^{1}$ Professor do Departamento de Clínica e Cirurgia Veterinária, Universidade Federal do Piauí, Teresina, Brasil. \\ ${ }^{2}$ Graduando em Medicina Veterinária, Universidade Federal do Piauí, Teresina, Piauí, Brasil. \\ ${ }^{3}$ Residente em Clínica e Cirurgia de Cães e Gatos, Hospital Veterinário da Universidade Federal do Piauí, Teresina, Brasil. \\ ${ }^{4}$ Residente em Anestesiologia Veterinária, Hospital Veterinário da Universidade Federal do Piauí, Teresina, Brasil. \\ *Autor para correspondência: flimavet@hotmail.com
}

\begin{abstract}
RESUMO. O monitoramento dos sinais vitais dos animais é essencial durante procedimentos de anestesia e cirurgia sendo a frequência cardíaca (FC) um dos parâmetros mais avaliados e para isso é necessário que se utilize métodos confiáveis. Com o objetivo de avaliar acurácia de dois métodos de aferição da FC em cães, realizouse estudo comparativo de dois equipamentos utilizados para esse fim, sendo estes equipamentos um medidor oscilométrico (Petmap $\left.{ }^{\circledR}\right)$ e um monitor cardíaco multiparamétrico (Cardiovet). Foram avaliadas 71 cadelas jovens e saudáveis sob anestesia geral inalatória, durante o procedimento cirúrgico de ovariossalpingohisterectomia eletiva com isofluorano. A partir do início de cada cirurgia, que durou em média 30 minutos, os valores da frequência cardíaca foram mensurados por ambos os aparelhos a cada 10 minutos, perfazendo um total de três medições por animal. Calculou-se então a média dos três valores aferidos e utilizou-se a média para avaliação por estatística descritiva e teste $\mathrm{t}$ de student. Os resultados para o equipamento oscilométrico e monitor cardíaco foram, respectivamente, 106,35 e 108,56 bpm, que não diferiram estatisticamente entre si. Isso mostra a semelhança da acurácia dos dois equipamentos na aferição da frequência cardíaca de cães, indicando que a escolha do equipamento pode ficar a cargo do médico veterinário, de acordo com a sua necessidade na clínica médica e cirúrgica, uma vez que o equipamento oscilométrico mede também a pressão arterial e o monitor cardíaco fornece adicionalmente dados eletrocardiográficos.
\end{abstract}

Palavras chave: analgesia, frequência cardíaca, monitoramento, oscilometria.

\section{Comparative study of heart rate obtained by oscillometric device and multiparameter monitor in female dogs during ovariohysterectomy}

\begin{abstract}
Monitoring of vital signs of animals is essential during anesthesia procedures and surgery being the heart rate (HR) one of the parameters evaluated and it is necessary to use reliable methods. Aiming to evaluate the accuracy of two methods of measuring HR in dogs, there was a comparative study of two devices used for this purpose, these being an oscillometric measuring equipment (Petmap $\left.{ }^{\circledR}\right)$ and a heart monitor multiparameter (Cardiovet). 71 healthy young bitches were evaluated under inhalation anesthesia during surgery elective ovariohysterectomy with isoflurane. From the beginning of the surgery, which lasted 30 minutes on average, the values of heart rate were measured by both devices every 10 minutes, for a total of three measurements per animal. We calculated the average of the three measured values and used the average for descriptive and evaluation by the Student $t$ test statistic. The results for the oscillometric machine and heart monitor were respectively 106.35 and $108.56 \mathrm{bpm}$, not statistically
\end{abstract}


different. This shows the similarity of the accuracy of the two devices in measuring the heart rate of dogs, indicating that the choice of equipment can be charged to the veterinarian, according to your need in the medical and surgical clinic, since the oscillometric device measures well as blood pressure and heart monitor additionally provides electrocardiographic data.

Keywords: analgesia, heart rate, monitoring, oscillometry

\section{Introdução}

Anestesia refere-se ao bloqueio ou remoção temporária dos mecanismos de dor através do uso fármacos com tal finalidade, que permitem a manipulação do paciente sem que o mesmo apresente sinais de dor ou que a tenha de forma amenizada (Fishbein, 1974). O controle álgico tem como objetivo fazer com que a dor seja mínima ou mesmo ausente nos procedimentos cirúrgicos, amenizar o sofrimento do paciente e permitir que este retorne o mais breve possível a sua normalidade (Slatter, 2007).

O tratamento pré-cirurgico, também designado como analgesia preemptiva visa uma analgesia pós-cirúrgica com redução da "memória de dor" (Alves et al., 2001). A redução do uso de fármacos anestésicos e manutenção do plano anestésico ideal também são promovidos pela analgesia preemptiva (Brondani et al., 2003). A chamada analgesia balanceada deve ser promovida por uma combinação de fármacos em doses ideais e via de administração adequada, no entanto a combinação perfeita de fármacos ainda é desconhecida (Slatter, 2007).

O procedimento cirúrgico para esterilização de fêmeas na medicina veterinária é denominado ovariossalpingo-histerectomia (OSH) e é frequentemente realizado com o intuito de controlar a população animal e evitar prováveis acometimentos patológicos do sistema reprodutor. Nas cadelas a analgesia para esse procedimento é obtida pelo uso de opioides, AINES e anestésicos locais, culminando com um efeito analgésico aditivo ou sinérgico (McQuay, 1992, Seli et al., 2005).

\section{Fármacos utilizados para ovariossalpingo- histerectomia}

\section{Acepromazina}

Fármaco pertencente ao grupo das fenotiazinas, com ação tranquilizante, que potencializa o efeito dos anestésicos gerais e que não produz analgesia em seu uso isolado (Birchard \& Sherding, 2008). Atua sobre o sistema nervoso central, causando depressão do tálamo e hipotálamo, promovendo assim a tranquilização e redução da atividade motora (Hall, 1985). Seu uso requer atenção, já que tem como efeito adverso o bloqueio alfa-adrenérgico que provoca hipotensão dose dependente e inibição plaquetária (Nunes et al., 1995, Birchard \& Sherding, 2008).

\section{Tramadol}

Opioide sintético derivado da codeína que atua sobre o sistema nervoso central promovendo analgesia (Aleixo \& Tudury, 2007). Possui ação analgésica em dor aguda e crônica pela ação sinérgica entre mecanismos opioides e não opioides (Santos et al., 2007). O tramadol pode ser responsável por pequenas alterações respiratórias, cardiovasculares e efeitos mínimos no peristaltismo gastrintestinal. Seu uso é efetivo no período pós-operatório de cirurgias ortopédicas, ginecológicas ou mesmo aplicações não cirúrgicas (Grond et al., 1995).

\section{Enrofloxacina}

Antimicrobiano de amplo espectro, pertencente ao grupo das fluorquinolonas (Mengozzi et al., 1996). Seu uso é permitido numa variedade de espécies animais, tornando este um antimicrobiano muito promissor na Medicina Veterinária, no entanto os cuidados quanto ao seu uso não fogem ao demais antimicrobianos, devido a possibilidade da ocorrência de resistência (Otero et al., 2001).

\section{Meloxican}

Anti-inflamatório não esteroidal (AINE), inibidor da COX-2 com vantagem sobre os AINEs não seletivos por não apresentar efeitos adversos que são provocados pela ação enzimática não seletiva. É absorvido em todas as espécies animais e tem melhor aceitação pela mucosa gástrica (Carneiro et al., 2014). Apresenta um tempo de recuperação que não varia conforme a quantidade de doses utilizadas ou mesmo com administração por infusão 
continua (Birchard \& Sherding, 2008). Apresenta ainda ação antioxidante sobre células dos rins, coração, cérebro e fígado (De La Cruz et al., 1998).

\section{Propofol}

Agente hipnótico, de uso intravenoso, de duração muito curta e recuperação rápida e suave. Promove perda da consciência entre 20 a 40 segundos após a sua aplicação intravenosa, por ser altamente lipofílico e assim rapidamente atravessar a barreira hematoencefálica (Duke, $\underline{1995)}$.

\section{Isofluorano}

Anestésico inalatório que necessita de inalação de uma concentração de 1 a $3 \%$ para que determine a indução anestésica (Birchard \& Sherding, 2008). Considerado segura, apresenta indução e recuperação rápidas (Massone, 1999). É o anestésico inalatório que provoca menor depressão do miocárdio, sendo assim recomendado para pacientes portadores de doença metabólica, geriátricos e susceptíveis a arritmias cardíacas (Birchard \& Sherding, 2008).

\section{Material e Métodos}

Foram avaliadas 71 cadelas, saudáveis e jovens durante a realização de ovariossalpingohisterectimia (OSH) eletiva. Todas as cadelas apresentavam peso entre $10 \mathrm{e}$ $20 \mathrm{~kg}$. Durante a preparação para o procedimento cirúrgico os animais foram submetidos a jejum alimentar de seis horas e hídrico de 2 horas e seguido ao jejum foram submetidos à préanestesia que envolveu acepromazina $0,1 \mathrm{mg} / \mathrm{kg}$ por via intramuscular (IM) para sedação e tranquilização, tramadol $2 \mathrm{mg} / \mathrm{kg}$ IM como o opioide eleito para propiciar analgesia desejada, enrofloxacina $10 \mathrm{mg} / \mathrm{kg}$ em via subcutânea (SC) prevenindo possíveis infecções perioperatórias, e meloxicam $0,2 \mathrm{mg} / \mathrm{kg}$ IM como para o controle da inflamação conseguinte à agressão cirúrgica aos tecidos. Para indução anestésica, administrou-se o propofol na dose efeito de $5 \mathrm{mg} / \mathrm{kg}$ em via intravenosa (IV). As cadelas foram intubadas com sonda orotraqueal de diâmetro adequado ao peso e, assim, conectadas através da mesma ao circuito semi-fechado com oxigênio a $100 \%$ para manutenção da anestesia inalatória efetivada por isofluorano (Figura 1).

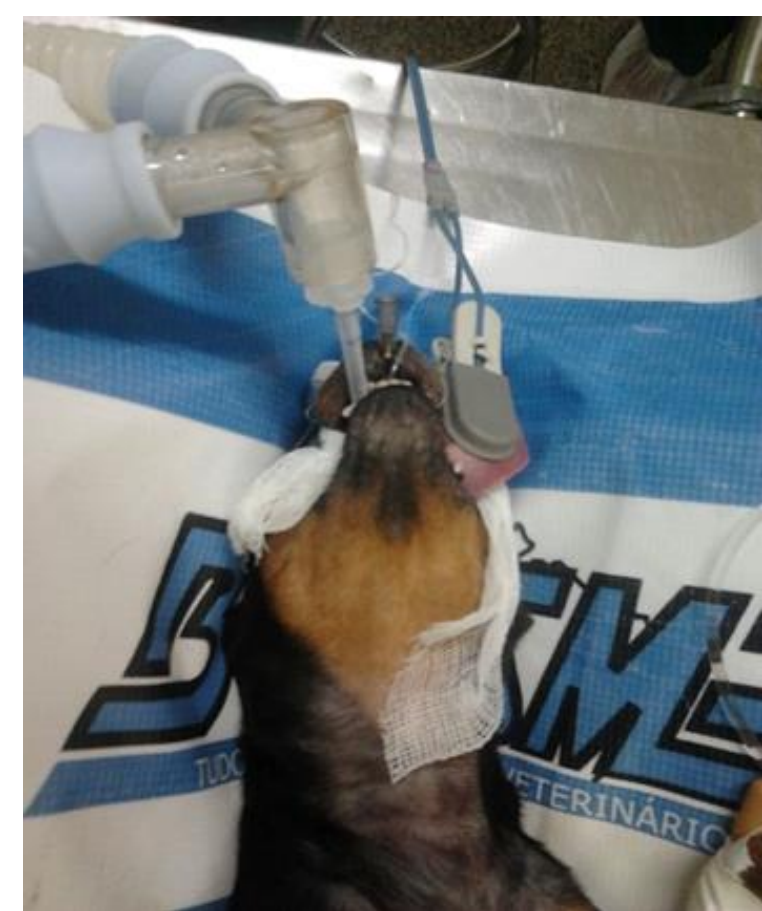

Figura 1. Paciente recebendo anestesia inalatória

A monitoração da frequência cardíaca (FC) foi realizada através de dois métodos distintos: aparelho medidor oscilométrico (Petmap $\left.{ }^{\circledR}\right)$ (Figura 2), e aparelho monitor multiparamétrico (Cardiovet) (Figura 3).

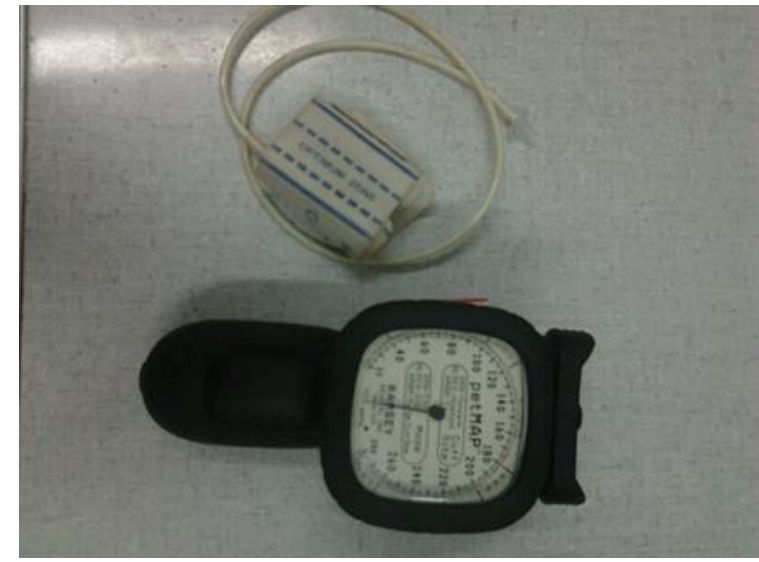

Figura 2. Medido Oscilométrico Petmap®

Os valores da FC foram mensurados por ambos os aparelhos durante o procedimento cirúrgico em intervalo de 10 minutos a partir do início do mesmo. Cada cirurgia durou em média 30 minutos, sendo aferidos, portanto, três valores de FC em cada paciente. Foi calculada a média dos três valores aferidos para cada animal, sendo as respectivas médias submetidas à análise estatística (Tabela 1), onde duas estatísticas descritivas e testes $\mathrm{F}$ foram realizados para comparar as variâncias dos dois aparelhos. 
Também foi aplicado Teste $\mathrm{T}$ para comparação entre as médias de cada animal obtidas para o Petmap ${ }^{\circledR}$ e as médias de cada animal obtidas para o monitor multiparamétrico.

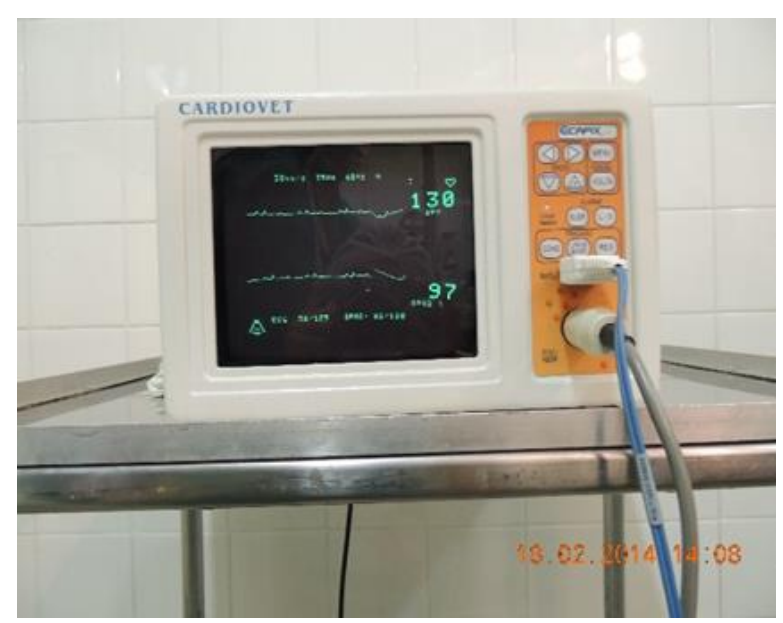

Figura 3. Monitor Multiparamétrico

\section{Resultados e discussão}

A análise estatística constatou a não significância do resultado no teste $\mathrm{F}$, sob nível de $5 \%$ de significância $(\mathrm{P}<0,05)$, indicando a igualdade da variância dos dois aparelhos para frequência cardíaca (FC). O Teste $\mathrm{T}$ efetuado para comparação entre as médias de cada animal obtidas para o Petmap® e as médias de cada animal obtidas para o monitor multiparamétrico também obteve resultado não significativo $(\mathrm{P}<0,05)$, indicando a igualdade estatística entre as duas médias (Tabela 1).

O monitor multiparamétrico geralmente é destinado a acompanhar a atividade cardíaca do pacienteavaliando os parâmetros vitais, principalmente a frequência cardíaca, no acompanhamento dos pacientes anestesiados, no entanto os dispositivos oscilométricos, como o Petmap ${ }^{\circledR}$ são práticos no quesito transporte, facilidade de manuseio, por ser um método automatizado, que possui um manguito pneumático que é inflado ao redor do membro até atingir a pressão suprassistêmica e este através de um microcomputador interno calcula e disponibiliza os valores de pressão arterial sistólica, diastólica, média e frequência cardíaca (Branson et al., 1997, Pierin \& Mion Júnior, 2001)

Tabela 1. Análise descritiva da comparação entre médias de frequência cardíaca (FC) obtidas de 71 cadelas (N) a partir do Petmap ${ }^{\circledR}$ (AP1) e do monitor cardíaco multiparamétrico (AP2), sob nível de 5\% de significância $(\mathrm{P}<0,05)$.

\begin{tabular}{cccccccc}
\hline Variável & $\mathrm{N}$ & Média & Mínimo & Máximo & $\mathrm{CV}$ & Variância & DP \\
\hline FC AP1 & 71,00 & 106,35 & 70,00 & 156,00 & 15,18 & 2260,66 & 16,15 \\
FC AP2 & 71,00 & 108,56 & 70,00 & 180,00 & 18,68 & 4211,34 & 20,28 \\
\hline
\end{tabular}

De modo geral, os equipamentos oscilométricos determinam as pressões arteriais sistólica, diastólica, média e a frequência do pulso (Brown \& Henik, 2002), e ainda arritmias produzidas pelos alguns fármacos utilizados na anestesia. No entanto, Slatter (2007) afirma que os dispositivos oscilométricos geralmente não funcionam bem nos casos de arritmias graves, bradicardia profunda ou taquicardia.

Para avaliações durante procedimentos cirúrgicos o monitor multiparamétrico apresenta uma eficiência melhor por estar conectado ao paciente constantemente, necessitando de manuseio mínimo e assim podendo demonstrar continuamente as alterações nos valores de frequência cardíaca. Porém, Jones (1996) salienta que a obtenção de dados referentes a parâmetros cardiovasculares depende da qualidade de ritmo cardíaco e pulso para que se realize medidas adequadas, corroborando assim com Carroll (1988), que afirmam que alguns monitores não indicam limites de referência para pequenos animais, podendo assim prejudicar sua utilização.

Alguns autores afirmam que o Petmap ${ }^{\circledR}$ é uma técnica razoavelmente precisa em cães de médio e grande porte, no entanto não recomendam seu uso em cães de pequeno porte ou gatos, justificando que nestes pacientes o aparelho tende a subestimar os valores de pressão arterial (Carvalho, 2009, Slatter, 2007). É importante lembrar que no presente estudo os animais em sua totalidade são de porte médio.

O monitor multiparamétrico apresenta uma facilidade de avaliação dos parâmetros referidos que depende do modelo a ser utilizado. $\mathrm{O}$ utilizado para esse trabalho pode ser mais 
trabalho devido a necessidade de fixar cabos com eletrodos em locais específicos da pele do animal, quanto que o Petmap ${ }^{\circledR}$ apenas um manguito é fixado em membro do animal, que determina a FC através do pulso, corroborando com Silva (2010) que concluiu que seu estudo que pela maior facilidade de manuseio e mensuração, o método oscilométrico é o mais adequado para uso na rotina clínica veterinária.

\section{Conclusão}

A não significância da comparação entre os resultados obtidos dos métodos de avaliação da frequência cardíaca em cadelas submetidas a ovariossalpingohisterectomia, demonstra que os dois aparelhos podem ser utilizados seguramente por oferecer precisão de forma semelhante, reservando ao médico veterinário a decisão, considerando as facilidades e dificuldades de manuseio de cada aparelho, de acordo com sua preferência na rotina das clínicas médica e cirúrgica, o método de monitoração a ser utilizado.

\section{Referências Bibliográficas}

Aleixo, G. A. S. \& Tudury, E. A. 2007. Utilização de opióides na analgesia de cães e gatos. Veterinária Notícias, 11, 31-42.

Alves, A. S., Campello, R. A. V., Mazzanti, A., Alievi, M. M., Faria, R. X., Stedile, R. \& Braga, F. A. 2001. Emprego do antiinflamatório não esteróide ketoprofeno na analgesia preemptiva em cães. Ciência Rural, $31,439-444$.

Birchard, S. J. \& Sherding, R. G. 2008. Manual Saunders: clínica de pequenos animais, São Paulo.

Branson, K. R., Wagner-Mann, C. C. \& Mann, F. A. 1997. Evaluation of an oscillometric blood pressure monitor on anesthetized cats and the effect of cuff placement and fur on accuracy. Veterinary Surgery, 26, 347-353.

Brondani, J. T., Natalini, C. C., Schossler, J. E. W., Pinto Filho, S. T. L. \& Bertin, A. P. 2003. Alterações cardiovasculares de gatos submetidos à toracotomia intercostal, prémedicados com associação de tramadol, butorfanol e atropina e anestesiados com propofol e halotano. Ciência Rural, 33, 869873.

Brown, S. A. \& Henik, R. A. 2002. Hipertensão sistêmica. In: Tilley, L. P., Goodwin, J.-K.,
Fagliari, J. J., Fagliari, G. S., Costa, M. T., Andrade, J. N. B., Rezende, M. L. \& Silva, N. N. (eds.) Manual de cardiologia para cães $e$ gatos. Roca, São Paulo.

Carneiro, S. C. M. C., Fonseca-Alves, C. E., Vicente, I. S. T., Martins, A. F. \& Fioravanti, M. C. S. 2014. Efeitos do meloxicam sobre as avaliações hematológica e bioquímica renal de cães adultos sadios. Revista de Ciência Veterinária e Saúde Pública, 1, 01-07.

Carroll, G. C. 1988. Blood pressure monitoring. Critical care clinics, 4, 411-434.

Carvalho, V. L. A. B. 2009. Hipertensão arterial felina. Faculdade de Medicina e Veterinária. Universidade Técnica de Lisboa, Lisboa.

De La Cruz, J. P., Sedeno, G., Carmona, J. A. \& De la Cuesta, F. S. 1998. The in vitro effects of propofol on tissular oxidative stress in the rat. Anesthesia \& Analgesia, 87, 1141-1146.

Duke, T. 1995. A new intravenous anesthetic agent: propofol. The Canadian Veterinary Journal, 36, 181-183.

Fishbein, M. 1974. The new illustrad medical and health encyclopedia. Home Library Edition, New York.

Grond, S., Meuser, T., Zech, D., Hennig, U. \& Lehmann, K. A. 1995. Analgesic efficacy and safety of tramadol enantiomers in comparison with the racemate: a randomised, double-blind study with gynaecological patients using intravenous patient-controlled analgesia. Pain, 62, 313-320.

Hall, L. W. 1985. Premedication in canine anesthesia. Canine Practice, 12, 16-21.

Jones, J. L. 1996. Noninvasive monitoring techniques in anesthetized animals. Veterinary Medicine, 91, 326-336.

Massone, F. 1999. Anestesiologia veterinária. Guanabara Koogan, Rio de Janeiro.

McQuay, H. J. 1992. Pre-emptive analgesia. British Journal of Anaesthesia, 69, 1-3.

Mengozzi, G., Intorre, L., Bertini, S. \& Soldani, G. 1996. Pharmacokinetics of enrofloxacin and its metabolite ciprofloxacin after intravenous and intramuscular administrations in sheep. American Journal of Veterinary Research, 57, 1040-1043.

Nunes, N., Pompermayer, L. G., Pirolo, J. \& Rahal, S. C. 1995. Emprego do metaraminol 
no bloqueio da hipotensão produzida pelo uso da levomepromazina em cães. Brazilian Journal of Veterinary Research and Animal Science, 32, 120-124.

Otero, J. L., Mestorino, O. N. \& Errecalde, J. O. 2001. Enrofloxacina: una fluorquinolona de uso exclusivo en veterinaria. Analecta Veterinaria, 21, 31-41.

Pierin, A. M. G. \& Mion Júnior, D. 2001. O impacto das descobertas de Riva-Rocci e Korotkoff. Revista Brasileira de Hipertensão, 8, 181-189.

Santos, A. C. D., Sousa, A. B., Florio, J. C. \& Spinosa, H. S. 2007. Estudo dos efeitos analgésicos e das concentrações plasmáticas do tramadol em cadelas submetidas a ováriosalpingo-histerectomia. Acta Scientiae Veterinariae, 35, 339-341.
Seli, K. A., Ana, R., Kamaja, O. \& Butinar, J. 2005. Intra-e efeitos analgésico pósoperatórios de carprofen em medetomidine premedicated dogs que sofre ovariectomy. Acta Veterinária (Beograd), v.55, n.5-6, p.435-448, 2005. Acta Veterinariae, 55, 435448.

Slatter, D. H. 2007. Manual de cirurgia de pequenos animais. Manole, São Paulo.

\section{Article History:}

Received 10 October 2016

Accepted 18 October 2016

Available on line 11 November 2016

License information: This is an open-access article distributed under the terms of the Creative Commons Attribution License 4.0, which permits unrestricted use, distribution, and reproduction in any medium, provided the original work is properly cited. 